\title{
In type A aortic dissection repair, an effective team approach and relational coordination are more important for patients' outcomes than surgeon volume
}

\author{
Ourania Preventza, MD, FACS
}

\footnotetext{
From the Division of Cardiothoracic Surgery, Michael E. DeBakey Department of Surgery, Baylor College of Medicine; and the Department of Cardiovascular Surgery, Texas Heart Institute, Houston, Tex.

Disclosures: O.P. consults for Medtronic, Inc, and participates in clinical trials for WL Gore \& Associates. In the past, she has received travel expenses from both Cook, Inc, and WL Gore \& Associates.

Received for publication Feb 10, 2017; accepted for publication Feb 15, 2017; available ahead of print March 25, 2017.

Address for reprints: Ourania Preventza, MD, FACS, Division of Cardiovascular Surgery, Texas Heart Institute, One Baylor Plaza, Houston, TX 77030 (E-mail: opsmile01@ aol.com).

J Thorac Cardiovasc Surg 2017;154:407-8

$0022-5223 / \$ 36.00$

Copyright (C) 2017 by The American Association for Thoracic Surgery

http://dx.doi.org/10.1016/j.jtcvs.2017.02.034
}

Whether elective or emergency cardiac surgical procedures performed by high-volume institutions or surgeons result in better outcomes is not entirely clear. Elegantly and with extensive statistical analysis, Bashir and colleagues ${ }^{1}$ have explored in the United Kingdom the surgeon volumeoutcome relation in patients treated for acute type A aortic dissection and concluded that a cardiac surgeon must have a minimum target case number of at least 4 to 4.5 cases annually to achieve significantly lower mortality than surgeons with a mean annual volume less than 4 .

This nicely written article raises more questions than answers. Do surgeons with 5 dissections under their belt and few or no elective aortic cases have better results than surgeons who have performed many elective arch cases but only 2 or 3 dissection repairs? Are outcomes the same among surgeons who have treated 4 dissections in a year but whose cumulative experience in elective aortic cases varies? I believe that the minimum number of cases that individual cardiac surgeons need to perform to significantly reduce the mortality associated with emergency aortic operations probably depends on their cumulative aortic experience.

The question becomes more complicated if we also consider hospital volume. Bashir and colleagues ${ }^{1}$ did not associate the hospital mean annual volume with the inhospital mortality rate.

Nobody would argue with the obvious: that high-volume aortic surgeons at high-volume institutions probably have better results than low-volume surgeons at low-volume institutions. But what happens with all the other possible combinations? Do low-volume surgeons at high-volume institutions have the benefit of treating more stable patients (who have been transferred from outside institutions), whereas low-volume surgeons at low-volume institutions treat sicker, less stable local patients? Are high-volume surgeons' outcomes the same at high- and low-volume hospitals? Could hospital volume, being an important

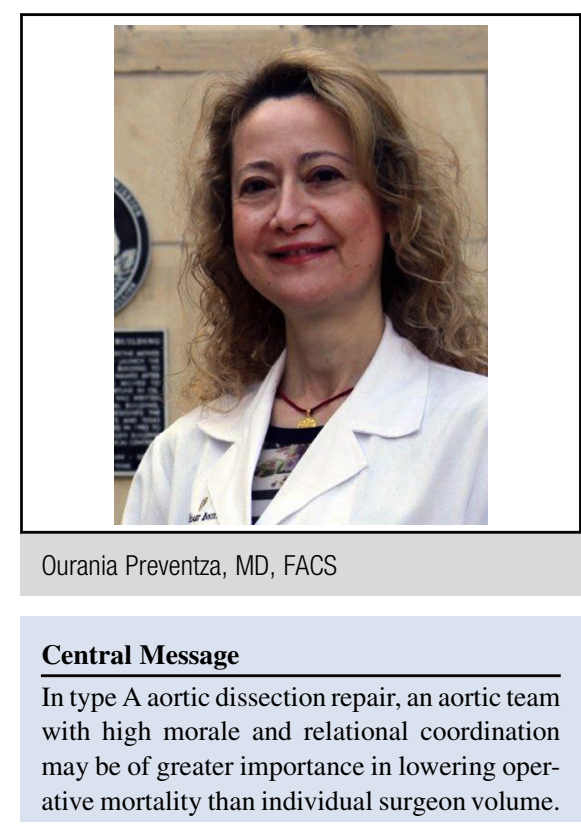

See Article page 398.

confounding factor, neutralize the effect of surgeon volume?

Furthermore, is a high-volume hospital more likely than a low-volume hospital to have an aortic surgical team? Among North American aortic centers, overall mortality improves after an aortic team using protocol-based management is established. ${ }^{2}$ Thus, although surgeon experience is extremely important in the treatment of acute type A aortic dissection, outcomes do not solely depend on the surgeon.

Achieving great results from a technically demanding operation requires team coordination from patient admission to discharge. This requires relational coordination among team members and experience doing the same or a similar task multiple times under elective circumstances, so that the urgency or difficulty of the situation does not alter the outcome disproportionally. The experience of the anesthesiologist; perfusionist; scrub, circulator, or intensive care unit nurse; intensivist; or the other surgeon involved in the case, and, most important, the relational coordination among all of them, could make or break a great result (Figure 1). Most of us, regardless of how much experience we have, feel relief when, in the middle of the night 


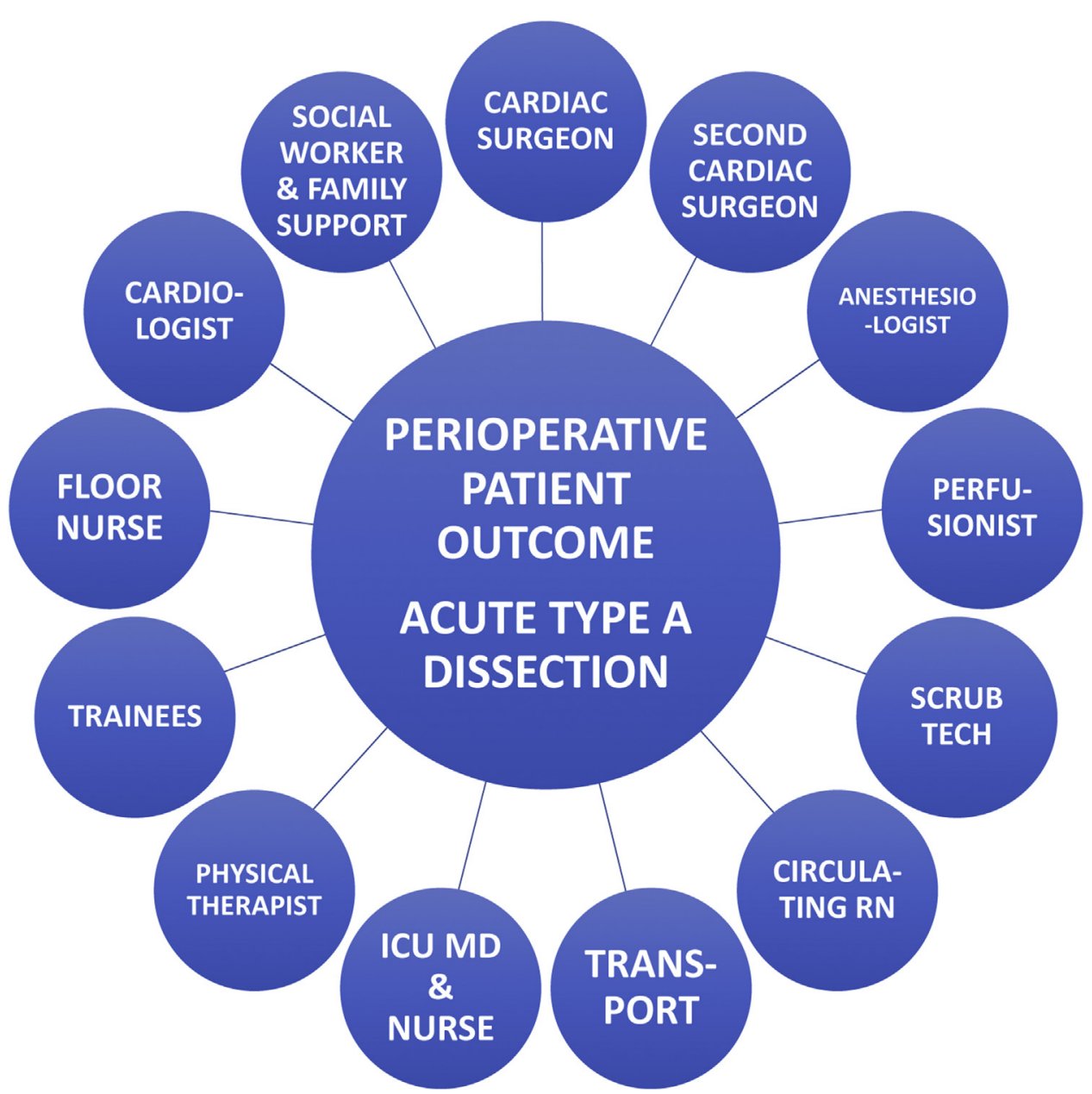

FIGURE 1. Achieving excellent outcomes depends more on relational coordination and perioperative team approach than on surgeon volume. (Figure does not include personnel involved in patient transfer between hospitals.) $I C U$, Intensive care unit.

or on a weekend, a surgical team member (anesthesiologist, scrub nurse, or fellow surgeon) familiar with aortic surgery shows up to help with a difficult case. Was it we, the experienced individual surgeons, who produced a great result, or was it the team that we had in addition to our experience? Without a doubt, I would choose a great team over a lone experienced individual. The task is to assemble such a team and, most important, to maintain the team's integrity and morale so as to deliver the desired results.

\section{References}

1. Bashir M, Harky A, Fok M, Shaw M, Hickey GL, Grant SW, et al. Acute type A aortic dissection in the United Kingdom: surgeon volume-outcome relation. $J$ Thorac Cardiovasc Surg. 2017;154:398-406.e1.

2. Preventza O, Coselli JS. Differential aspects of ascending thoracic aortic dissection and its treatment: the North American experience. Ann Cardiothorac Surg. 2016;5:352-9. 